\title{
A Passive Auto-switching Amphibian Antenna
}

\author{
Steven G. O'Keefe and Steven Perhirin
}

\begin{abstract}
An amphibian antenna was constructed that is capable of radiating both above and below the surface of water. The antenna is effectively a dual band PIFA design where each radiating element is tuned to resonate in close proximity of either air or water. A tuning stub was switched into circuit by the use of a passive, water filled, capacitive microstrip switch. The antenna was designed to operate at $433 \mathrm{MHz}$ and good input impedance was observed in both simulation and measurement for both environments.
\end{abstract}

Index Terms - Underwater antenna, microstrip switch, dual band PIFA.

\section{INTRODUCTION}

$\mathrm{W}$ IRELESS sensors and radio telemetry systems are now in common usage. Some of these networks or communication systems are intended to function underwater [1], in some cases under seawater [2]. There are some applications where the sensor node will cross from one medium to another. This could, for example, be a change from air to earth, or air to water. Examples of the latter might be monitoring the human body whilst engaged in activities that involve being both fully submerged and out of the water. Many sporting activities fall into this category [3]. Similarly control and communication with remote control vehicles can call for a wireless system which can function in both environments [4].

Water and air have very different electrical properties. Possibly the most significant difference being that of the relative permittivity $\left(\varepsilon_{\mathrm{r}}\right)$, with air being 1 and water being approximately 81 , dependent on temperature etc. When conductors carrying high frequency signals are immersed in a dielectric medium, it is well understood that the length of the conductor, in wavelengths, is increased by a factor approximately equal to $\sqrt{\varepsilon_{r}}$. This of course means that antennas designed for operation in air will seem 9 times longer, and exhibit completely different properties if submerged into water. Studies have shown that even if the submerged antenna is well insulated from the water, the resonant frequency will be significantly shifted [5].

An approach to solve this problem has been to design

Manuscript received June 20, 2013.

S. G. O'Keefe is with the Centre for Wireless Monitoring and Applications, Griffith School of Engineering, Griffith University, Nathan, Brisbane, QLD, Australia (e-mail: s.okeefe@griffith.edu.au).

S. Perhirin, was on internship at Griffith University from Universite de Bretagne Occidentale, Brest, France. (e-mail: steven.perhirin@gmail.com). wideband antennas that have sufficient bandwidth such that when shifted in frequency due to the surrounding water, will still have a usable input impedance at the frequency of interest $[6,7]$. Another solution to the problem could be to have two

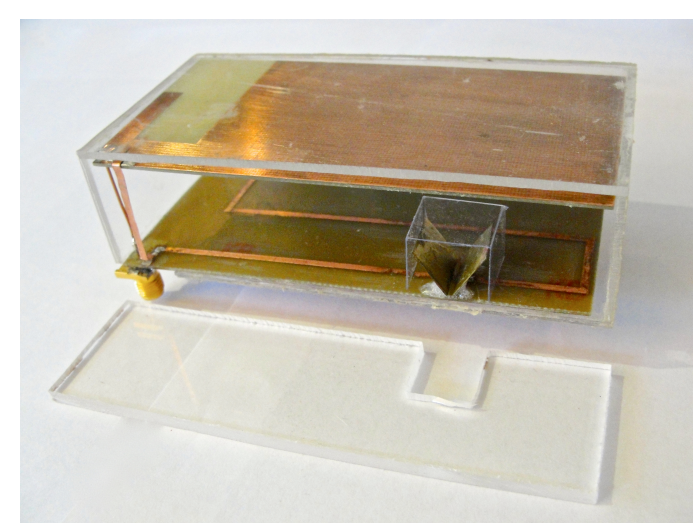

(a)

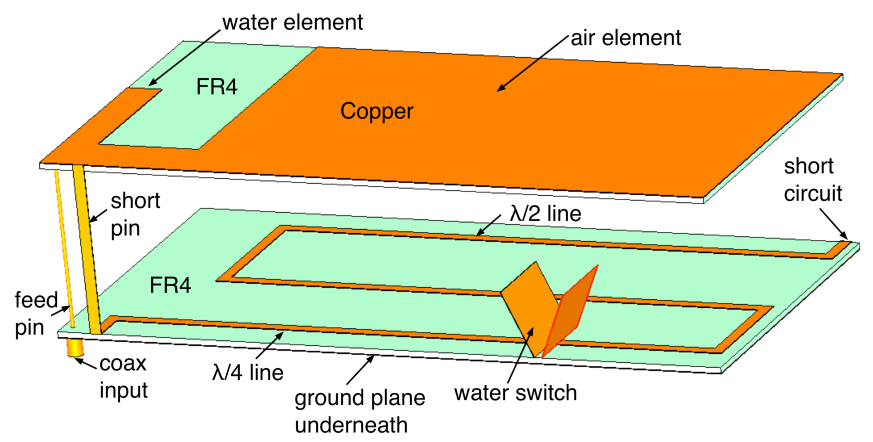

(b)

Fig. 1. (a) Photo of one of the prototype antennas prior to sealing of the waterproof housing and inlet port. (b) The design of the amphibian antenna.

separate antennas, and an RF switch under the control of a sensor, which could determine which media the antenna is in at that time. However a simpler solution would be to design a dual band antenna. Normally dual band antennas have two functional sections that will individually resonate at the two different design frequencies. In the case of the amphibian antenna the two frequencies are exactly the same, but the properties of the surrounding environment is changing with time. Thus we need a dual band antenna where both sections, or elements, are tuned to the same frequency, but one element is resonant in air and the other is resonant when immersed in water. Perhaps this antenna should more correctly be called a dual environment antenna. Both sections should be connected to the same feed-point in a similar manner to dual band antennas. This obviates the need for an RF switch. The applications for this antenna necessitate a compact and low cost solution, so this is a better solution. The frequency used for this work was $433 \mathrm{MHz}$. 


\section{AMPHIBIAN ANTENNA DESIGN}

A planar inverted $F$ antenna (PIFA) design was chosen for the amphibian antenna and is shown in the top PCB depicted in Fig. 1. A more optimum antenna may be chosen for specific applications and this study is mainly to demonstrate the passive switching technique. The dual environment functionality was incorporated by having two elements of different size, connected to the same feed-point. The radiating elements were printed on an FR4 PCB and placed over a groundplane, also constructed on FR4, with full copper on the underside, as shown (bottom PCB). The tuning circuit depicted on the upper side of the groundplane PCB will be discussed in section IV. The entire antenna (radiating elements and groundplane) was sealed inside a perspex box (shown in Fig. 1a) with internal dimensions of $48 \times 98 \times 25 \mathrm{~mm}$ and wall thickness of $1 \mathrm{~mm}$. As is often the case with a compact PIFA, the feed-point impedance needed correcting by the use of a shorting pin near the feed-point. In this case it was found that the element to be used underwater (water element) was effectively lengthened due to the high permittivity of the surrounding water. The input impedance presented by this element was not ideal $(50 \Omega)$, but it presented a usable impedance of $114+\mathrm{j} 43 \Omega$. The input impedance presented by the other element to be used in air (air element) was far from ideal at $3+\mathrm{j} 33 \Omega$ and did require the shorting pin for impedance matching. Fig. 2 shows the simulated input impedance plots for both water and air elements.

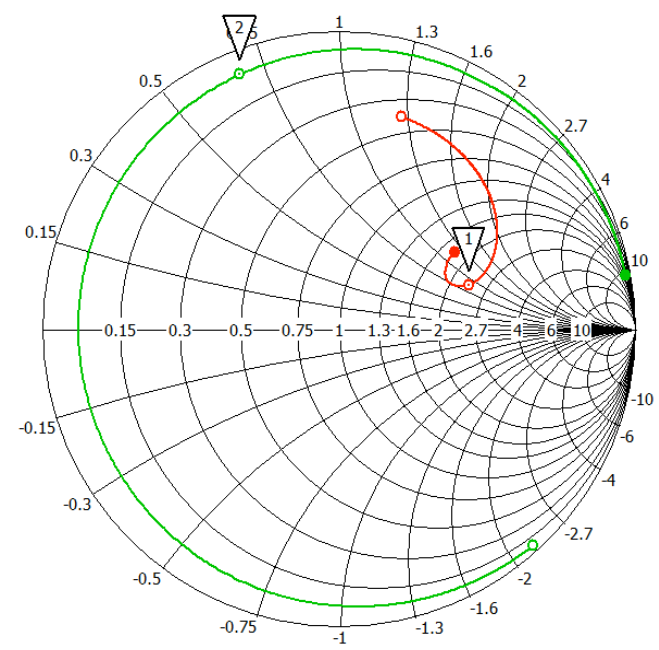

Fig. 2. Simulated input impedance of the water element (1) and air element (2) when input impedance is unmatched. Markers are at the design frequency $(433 \mathrm{MHz})$. Frequency range of traces are $200-800 \mathrm{MHz}$.

To solve this problem the impedance of the water element could be deliberately shortened and therefore detuned so that it also needed the short to correct its input impedance. This would however degrade the radiation efficiency of this element. An alternative solution was devised to passively switch the short in or out, dependent on whether the antenna was in air or water.

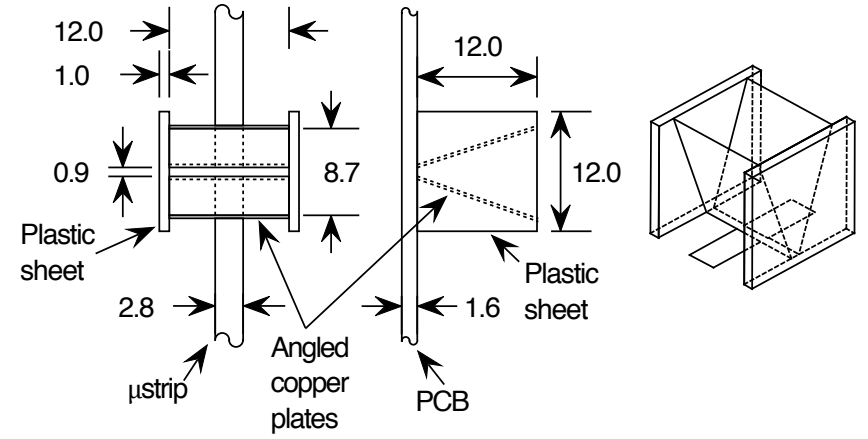

Fig. 3. The structure of the water activated switch.

\section{WATER ACTIVATED SWITCH}

To keep the antenna design simple and cheap to manufacture, a passive solution was explored to switch the impedance matching circuit in when required. Fig. 3 shows the structure of the switch. The two angled copper plates projecting from the plane of the PCB are capacitive plates. A microstrip line is connected to each plate. This switch can also be seen in Fig 1. When there is air between the plates the capacitance will be small and the reactance therefore large. Effectively there is an open circuit between the microstrip lines at this point in the circuit. When the volume between the plates is filled with water, the capacitance is much larger, thus creating a lower impedance path through the circuit. For the switch tests, the switch element was placed on an FR4 PCB of $100 \times 50 \mathrm{~mm}$ dimensions. SMA connectors were placed on the two short sides and $50 \Omega$ microstrip was used to connect through the switch element. Two pieces of $1 \mathrm{~mm}$ plastic sheet were used to seal off the ends of the angled copper plates in order to create a well for them to hold the water for the test.

Fig. 4 shows the simulated and measured results for the water activated switch alone. All simulations were done in CST Microwave Studio and all measurements were taken on a Rhode and Schwartz ZVRE vector network analyser. When the well is filled with water a meniscus forms at the top. This was also included in the simulation. The results show that when the switch does not contain water the measured transmission coefficient, at the frequency of interest (433 $\mathrm{MHz})$, is approximately $-13 \mathrm{~dB}$. The simulation shows a similar result. When fresh water was placed into the switch the coefficient dropped to $-0.7 \mathrm{~dB}$, with the simulation showing a similar result.

\section{AUTO-TUNED ANTENNA}

A water activated switch was now used to auto-tune the antenna. As stated earlier, when in air the antenna requires a short placed near the feed-point to match the impedance. To accommodate this a microstrip line was placed on the top side of the groundplane PCB. This line connects to the shorting pin coming from the radiating element on the top PCB (see Fig 1.), and in conjunction with the length of the shorting pin, has a length of approximately $1 / 4 \lambda$. Both PCBs are FR4 and are $0.8 \mathrm{~mm}$ thick and they are separated by $24 \mathrm{~mm}$. The end of this 

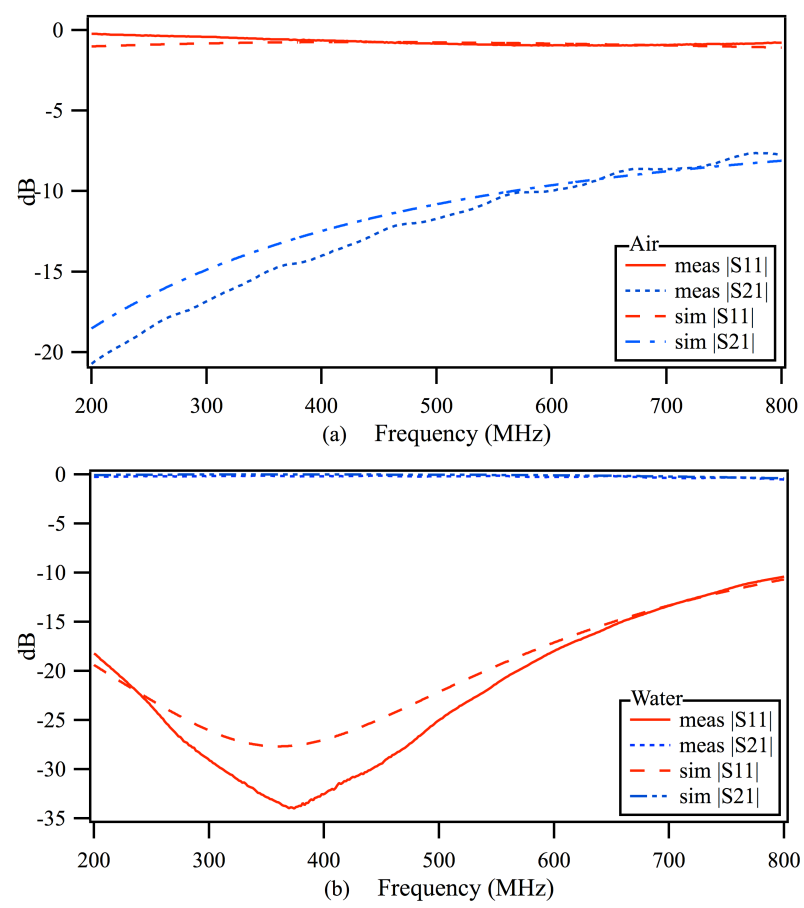

Fig. 4. Simulated and measured $\left|S_{11}\right|$ and $\left|S_{21}\right|$ results for the water activated switch in both (a) air and (b) water.

$1 / 4 \lambda$ line is terminated in the water switch. The copper plates of the water switch implemented on this design were $9.85 \times 12$ $\mathrm{mm}$ in size and are placed at 24 degrees to the normal to the PCB. Fig. 5 shows the structure. The angle of the copper plates can be varied and a smaller angle gives greater capacitance across the switch. In this case the angle was chosen so that water would easily enter and leave the space between the plates via the hole in the waterproof case. With a smaller angle, the surface tension impedes this action. The size and separation of the plates is chosen such that the reactance difference, when filled with water or air, gives the desired $\left|S_{21}\right|$ values. When there is air between the plates of the switch, the shorting pin together with the line will be approximately one

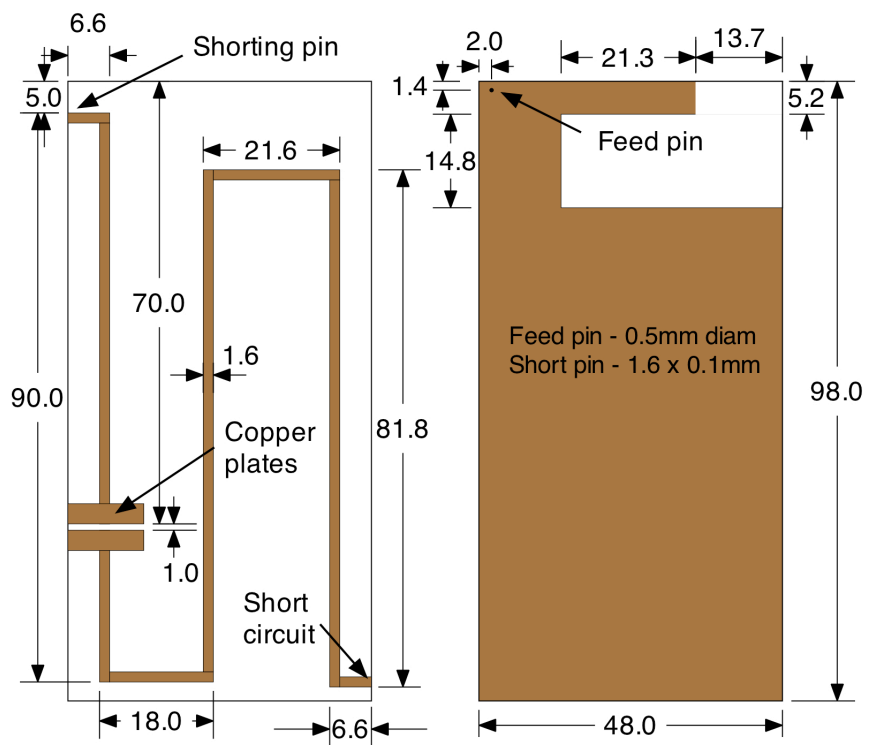

Fig. 5. Dimensions of the tuning stub, water switch and PIFA element for the amphibian antenna. quarter wave in length and will resemble a short circuit to ground, and thus will show a very low impedance at the feed point.

The far side of the water switch is connected to another microstrip line, which is approximately $1 / 2 \lambda$ long and terminated in a short circuit. When the water switch is filled with water the switch effectively connects the two microstrips together making a line of approximately $3 / 4 \lambda$ in length. The input impedance of this combination means the shorting pin from the antenna element actually appears to be an open circuit from its input end. One might imagine that this could be more easily accomplished by placing a short circuit directly after the water switch, thus providing an open circuit at the input of the $1 / 4 \lambda$ line. However when the antenna is submerged the shorting pin between groundplane and radiating element is partially surrounded by water. This increases its electrical length, which makes the length of the line between shorting pin and water switch longer than $1 / 4 \lambda$.

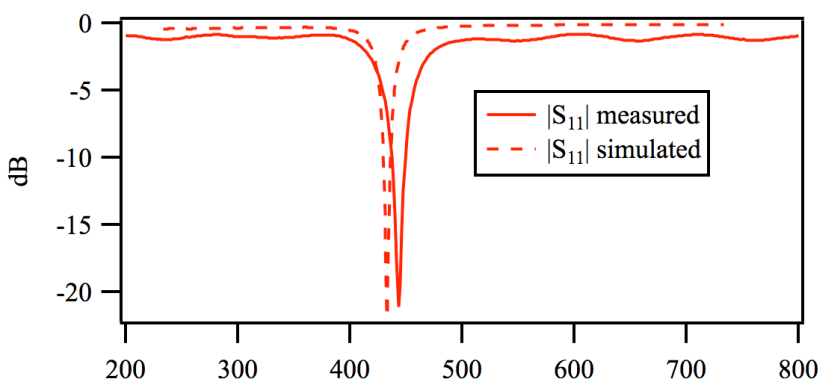

(a) Frequency $(\mathrm{MHz})$

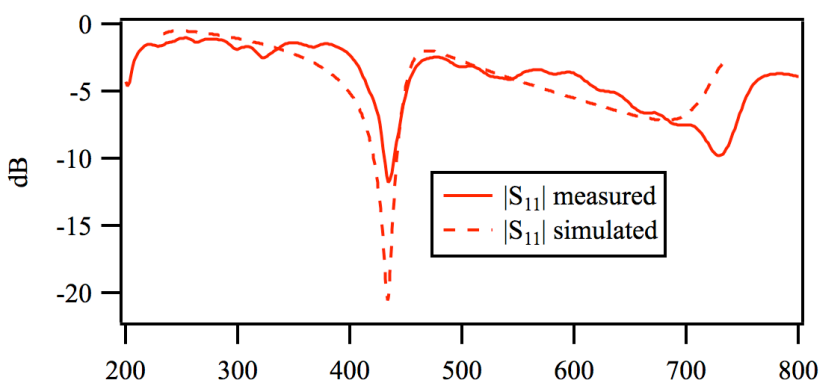

(b) Frequency $(\mathrm{MHz})$

Fig. 6. Simulated and measured $\left|S_{11}\right|$ results for the complete amphibian antenna in both (a) air and (b) water.

The water switch was sealed off from the rest of the antenna to avoid flooding the remainder of the antenna volume, and a hole was included in the outside of the antenna's perspex box to allow water to enter when the antenna was submerged. The complete antenna was simulated and a prototype was tested both in air and submerged in tap water. Fig. 6 shows the results. There is good correlation between measured and simulated results and the match is good at band centre. The simulated radiation efficiency (not including mismatch losses) of the antenna was $63 \%$ in water and $36 \%$ in air. Fig. 7 shows both the radiation efficiency and total efficiency (including mismatch losses) around the design frequency of $433 \mathrm{MHz}$. These figures are realistic given the compact nature of the antenna. The simulated efficiency of the antenna in water is 


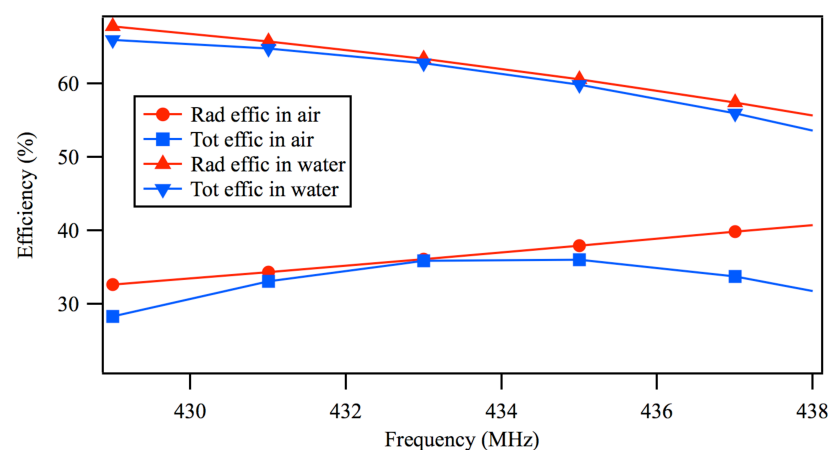

Fig. 7. Simulated radiation and total efficiency for the complete amphibian antenna immersed in both both air and water.

higher than air. The close proximity of the water to the radiating element, ground plane and region between the two, when the antenna is submerged, will provide dielectric loading and significantly increase the electrical dimensions, and thus volume, of the water element. This increase in dimension of an electrically small antenna can increase the efficiency of an antenna. The expected reduction in bandwidth due to this loading is offset by the loss of the loading material, in this case the water. In this case the loading material is also the propagation media. The dependence of the efficiencybandwidth product due to dielectric loading has been demonstrated earlier [8]. Also the very low unmatched impedance of the air element may contribute to its poorer efficiency. The measured $-6 \mathrm{~dB}$ impedance bandwidth is $25.5 \mathrm{MHz}$ in water and $22 \mathrm{MHz}$ in air. The intended application for this antenna only has a required bandwidth of less than $100 \mathrm{kHz}$.

The total amphibian antenna design realizes an antenna that can passively detect the presence of water or air around the antenna and switch the appropriate matching element into circuit. The bandwidth and efficiency of the current design is adequate for many applications, but could be improved by utilizing wider bandwidth antenna designs and wider bandwidth matching elements, still incorporating the water switch technique. The current design relies upon water entering an open port on the side of the antennas waterproof enclosure. When the antenna is held at certain angles, with respect to the waters surface, the flow of water into and out of the port can be impeded. A refinement will be to reorient the metal plates making up the water switch such that they placed outside the waterproof enclosure. In this way water can freely flow between the plates.

The propagation loss through fresh water at these frequencies is still quite high, but many of the planned applications only require transmission over relatively short distances (a small number of metres). Assuming a conductivity $(\sigma)$ of around $0.01 \mathrm{~S} / \mathrm{m}$ for fresh water. The calculated attenuation constant $(\alpha)$ at $433 \mathrm{MHz}$ would be -13.5 $\mathrm{dB} / \mathrm{m}$ using

$$
\alpha=20 \log \left(\frac{\sigma}{2} \sqrt{\frac{\mu}{\varepsilon}}\right)
$$

where $\mu$ and $\varepsilon$ are the permeability and permittivity respectively. This gives a skin depth $(\delta)$ of $4.7 \mathrm{~m}$ where $\delta=1 / \alpha$.
Given modern sensitive receivers this makes communications possible in water at this frequency. One must of course realise that the divergence factor for a submerged point source transmitter, which is proportional to $1 /(\mathrm{kR})^{2}$, will also significantly affect signals as the wavenumber $(\mathrm{k})$ will be large due to the high permittivity of water. Reflection losses at the surface will also be significant, but the high angle of refraction, due to the large difference in permittivity between air and water, will be of advantage in directing transmissions close to parallel to the waters surface.

\section{CONCLUSIONS}

An amphibian antenna has been built which can function both in air and underwater by passively tuning itself. Tests so far have only been undertaken in fresh water and chlorinated pool water. Further work would be needed to see if a similar design, possibly at lower frequencies, could be feasible in much more conductive seawater.

\section{REFERENCES}

[1] C. Xianhui, I. Wells, G. Dickers, P. Kear and G. Xiaochun, "Reevaluation of RF electromagnetic communication in underwater sensor networks," IEEE Communications Magazine, Dec 2010, pp. 143-151.

[2] M. Rhodes, "Electromagnetic propagation in sea water and its value in military systems," SEASDTC Proceedings of the second annual conference 2007,

http://www.seasdtc.com/events/2007_conference/mission_planning_and decision_making.htm

[3] D. A. James, N. Davey and T. Rice, "An Accelerometer Based Sensor Platform for Insitu Elite Athlete Performance Analysis," Proceedings of IEEE Sensors 2004, vol 3, pp. 1373-1376.

[4] T. Shaneyfelt, A. Joordens, K. Nagothu and M. Jamshidi, "RF communication between surface and underwater robotic swarms," Proceedings of the World Automation Congress, 2008, pp. 1-6.

[5] D. A. James, A. Galehdar and D. V. Thiel, "Mobile sensor communications in aquatic environments for sporting applications," Procedia Engineering 2, 2010, pp. 3017-3022.

[6] A. M. Abbosh, D. A. James and D. V. Thiel, "Compact UHF antenna in aquatic environments for mobile sporting applications," Antennas and Propagation Society International Symposium, 2010, pp. 1-4.

[7] A. G. Miquel, "UWB antenna design for underwater communications," Masters thesis, Universitat Politècnica de Catalunya, 2009, http://hdl.handle.net/2099.1/7585.

[8] Ichirou Ida, Jin Sato, Takatoshi Sekizawa, Hiroyuki Yoshimura \& Koichi Ito, "Dependence of the Efficiency-Bandwidth Product on Electrical Volume of Small Dielectric Loaded Antennas." IEEE Transactions on Antennas and Propagation, Vol. 50, No. 6, June 2002. 